\title{
Validation of a dosing regimen for fixed-dose, weight- adjusted, subcutaneous unfractionated heparin for the acute treatment of venous thrombo-embolism in a population from a resource-constrained environment
}

\author{
J I Munsamy, H Kertland, A Parrish
}

To the Editor: A study in Canada and New Zealand (Kearon et al.) suggested that fixed-dose unmonitored subcutaneous (SC) unfractionated heparin (UFH) is as effective and safe as low-molecular-weight heparin (LMWH) for the acute treatment of venous thrombo-embolism (VTE). While this trial has limitations, it provides evidence to support the use of SC UFH in a resource-constrained environment. However, because public sector patients with VTE in South Africa often have multiple co-morbidities and are thinner and younger than those in that study, the local validity of the published dosing regimen is unclear.

\section{Background}

VTE is a potentially fatal disorder manifesting as deep-vein thrombosis (DVT) and pulmonary embolism (PE). ${ }^{1}$ Initial standard management of VTE was with intravenous UFH with dose adjustments according to the partial thromboplastin time (PTT). ${ }^{2}$ This regimen is effective, but is limited by the requirements for intensive monitoring and ensuring a constant heparin infusion rate. The introduction of SC LMWH using weight-based dosing offered an attractive alternative to UFH, with proven efficacy and safety. ${ }^{3}$ Although LMWH is more expensive, this cost is offset by reduced laboratory costs, as PTT monitoring is not required.

Kearon et al. performed a randomised, open-label, adjudicator-blinded trial (FIDO) suggesting that fixed-dose, weight-adjusted SC UFH, without PTT monitoring, appeared as effective and safe as fixed-dosed, unmonitored SC LMWH in patients with acute VTE. ${ }^{4}$ SC UFH was given as a loading dose of 333 units $/ \mathrm{kg}$ followed by 250 units $/ \mathrm{kg}$ 12-hourly. ${ }^{4} \mathrm{~A}$ recent review concluded that SC UFH appeared to be as effective and safe with regard to rates of bleeding and death. ${ }^{5}$ However, the authors declared that more robust data were needed to recommend SC UFH without PTT monitoring.

Although the use of LWMH is established, the considerably less costly approach of fixed-dose, weight-adjusted, SC UFH is appealing in a resource-constrained setting. However,

Doctor of Pharmacy Programme, Faculty of Pharmacy, Rhodes University, Grahamstown, E Cape

J I Munsamy, BPharm

H Kertland, PharmD

Associate Professor, Department of Medicine, Walter Sisulu University, Mthatha, E Cape

A Parrish, FCP (SA) there appears to be no published evidence to indicate that this regimen has been validated locally in patients who differ from those in the FIDO study in age, weight, and presence of multiple co-morbidities.

\section{Methods}

The protocol was approved by the East London Hospital Complex Research and Ethics Committee. The sample size was based on a previous study that looked at optimal dosing of SC UFH for the treatment of DVT. ${ }^{6}$ Patients provided written informed consent. Concurrent chart review was performed on all consecutive eligible patients (age $\geq 18$ years; newly diagnosed VTE) admitted between November 2008 and February 2009 to internal medicine wards at Cecilia Makiwane Hospital and who had been prescribed SC UFH according to the FIDO study. All other aspects of care were delivered as standard of care. The primary measure of interest was the proportion of patients with a therapeutic PTT (patient PTT 1.5 - 2.5 times the control value) measured approximately 6 hours after the morning dose of UFH on any day from the 2nd to the 6th day of SC UFH therapy as in the FIDO regimen. Neither safety nor efficacy of patient outcomes were collected as the sample size was chosen only to validate the published dosing regimen.

\section{Results}

A total of 25 inpatients were recruited of whom 24 had acute DVT confirmed on Doppler ultrasound, and one had clinical evidence of $\mathrm{PE}$ and a strongly positive d-dimer result. The mean age of the patients was 37.6 years (SD 15.03); mean body weight $57.2 \mathrm{~kg}$ (SD 16.79) and 68\% were female (Table I). Pulmonary tuberculosis was the most prevalent concomitant disease $(64 \%)$. The mean loading and maintenance doses of UFH were 320 units $/ \mathrm{kg}$ (SD 66.87) and 250 units/ $\mathrm{kg}$ (SD 5.57) respectively. PTT was measured on a single blood sample obtained a mean of 6.0 hours (SD 0.37) after the morning dose and a mean of 3.48 days (SD 0.92) after starting SC UFH. Twelve ( $48 \%$ ) of the 25 patients had a PTT within the therapeutic range, $8(32 \%)$ patients were found to have a supratherapeutic PTT (patient value $>2.5$ times control value) and $5(20 \%)$ had a sub-therapeutic PTT (patient value $<1.5$ times control value). The mean PTT ratio (PTT patient/PTT control) was 2.44 (SD 1.40; 95\% CI 1.89 to 2.99). The mean duration of heparin therapy was 8.5 days (SD 3.39).

\section{Discussion}

Baseline characteristics of patients in this study including gender, age and weight differed from the SC UFH treatment arm in FIDO (Table I). There were relatively more women than 


\section{SCIENTIFIC LETTERS}

Table I. Comparison between this study, FIDO and the dose validation study

\begin{tabular}{llll}
\hline & & \multicolumn{2}{c}{ Previous studies } \\
\cline { 3 - 4 } & This study & Dose validation $^{6}$ & FIDO $^{4}$ \\
\hline Baseline characteristics & & 20 & 355 \\
$\quad$ Number of patients & 25 & 67 & $60(17)$ \\
Age, mean (SD) years & $38(15)$ & 78 & $82(19)$ \\
Weight, mean (SD) kg & $57(17)$ & 60 & 49 \\
Women (\%) & 68 & & $37(19)$ \\
PTT (Day 3) & $12(48)$ & $12(60)$ & $39(20)$ \\
Therapeutic, No. (\%) & $8(32)$ & $4(20)$ & $39(20)$ \\
Supratherapeutic, No. (\%) & $5(20)$ & & \\
Sub-therapeutic, No. (\%) & &
\end{tabular}

men (68\% versus $49 \%$ in FIDO) and the mean ages and weights were less (38 years v. 60 years and $57 \mathrm{~kg} \mathrm{v.} 82 \mathrm{~kg}$ ).

None of our patients had previous VTE, compared with $11 \%$ of patients in the SC UFH group in FIDO. Sixteen patients were diagnosed with DVT and concomitant tuberculosis but none in the SC UFH group in FIDO.

In FIDO, the PTT (therapeutic PTT: 60 - 85 seconds) was measured in 197 patients, a mean of 6.0 hours after the morning dose and a mean of 2.8 days after starting $\mathrm{UFH}^{4}$ Thirty-seven patients (19\%) had a PTT within the therapeutic range, $121(61 \%)$ were found to be supratherapeutic $(>85$ seconds) and 39 (20\%) sub-therapeutic ( $<60$ seconds). ${ }^{4} \mathrm{~A}$ higher proportion of patients in our cohort appeared to have therapeutic values, while a lower proportion had supratherapeutic and a similar proportion sub-therapeutic values. The low sub-therapeutic ratio for 1 patient in our cohort may be attributed to 2 dose omissions prior to the blood samples taken for PTT monitoring.

\section{Conclusion}

In a small but real-world pragmatic study, the use of a weight-based dosing nomogram appeared feasible, and PTT results obtained were within the range achieved in a previous large trial comparing LMWH with fixed-dose, weight-based SC UFH. Our study provides preliminary support for the local generalisability of the FIDO dosing model, offering a promising alternative for the treatment of VTE in a resourceconstrained environment.

References

1. Haines ST, Zeolla M, Witt DM. Venous thromboembolism. In: Dipiro JT, Talbert RL, Yee GC, Matzke GR, Wells BG, Posey LM, eds. Pharmacotherapy: A Pathophysiologic Approach. 7th ed. Columbus, OH: McGraw-Hill Companies, 2008: 331-371.

2. Raschke RA, Reilly BM, Guidry JR, Fontana JR, Srinivas S. The weight-based heparin dosing nomogram compared with a "standard care" nomogram. Ann Intern Med 1993; 119: 874-881.

3. Van Dongen CJ, van den Belt AGM, Prins MH, Lensing AWA. Fixed dose subcutaneous low molecular weight heparins versus adjusted dose unfractionated heparin for venous thromboembolism. Cochrane Database of Systematic Reviews 2004, Issue 4. Art. No.: CD001100. DOI: 10.1002/14651858.CD001100.pub2.

4. Kearon C, Ginsberg JS, Julian JA, et al. Comparison of fixed-dose weight-adjusted unfractionated heparin and low-molecular weight heparin for acute treatment of venous thromboembolism. JAMA 2006; 296: 935-942.

5. Vardi M, Zittan E, Bitterman H. Subcutaneous unfractionated heparin for the initial treatment of venous thromboembolism. Cochrane Database of Systematic Reviews 2009, Issue 4. Art. No.: CD006771. DOI: 10.1002/14651858.CD006771.pub2.

6. Kearon C, Harrison L, Crowther M, Ginsberg JS. Optimal dosing of subcutaneous unfractionated heparin for the treatment of deep vein thrombosis. Thromb Res 2000; 97: 395403.

Accepted 11 May 2010. 